\title{
Delayed Paraplegia Triggered by Gastrointestinal Bleeding 8 Months after TEVAR: Persistent Vulnerability of Spinal Cord
}

\author{
Takashi Yamauchi, MD, PhD, Suguru Kubota, MD, PhD, and Kosei Hasegawa, MD
}

We report a rare case of delayed paraplegia triggered by gastrointestinal (GI) bleeding 8 months after thoracic endovascular aortic repair (TEVAR). A 78-year-old male underwent TEVAR of a descending thoracic aortic aneurysm without a postoperative neurological deficit and was discharged. Magnetic resonance image showed spinal cord infarction from Th8 to L1, and enhanced computed tomography showed a patent Adamkiewicz artery. The ostium of the intercostal artery connected with the Adamkiewicz artery was occluded. Patients with a history of TEVAR might be more vulnerable to spinal cord ischemia around the Adamkiewicz artery, which can be triggered by common hemorrhagic diseases, such as Gl bleeding, even remote from the procedure.

Keywords: thoracic endovascular aortic repair (TEVAR), delayed paraplegia, spinal cord ischemia

\section{Introduction}

Repair of descending thoracoabdominal aortic aneurysms is challenging because of possible catastrophic complications. Thoracic endovascular aortic repair (TEVAR) is expected to reduce the incidence of such complications. However, delayed paraplegia is well known to develop several hours to 1 week after surgery and has been re-

Department of Cardiovascular Surgery, KKR Sapporo Medical Center, Sapporo, Hokkaido, Japan

Received: July 31, 2018; Accepted: August 31, 2018

Corresponding author: Takashi Yamauchi, MD, PhD. Department of Cardiovascular Surgery, KKR Sapporo Medical Center, 6-3-40 Ichijo Hiragishi, Toyohira, Sapporo, Hokkaido 0620931, Japan

Tel: +81-11-822-1811, Fax: +81-11-841-4572

E-mail: yamataka@surg1.med.osaka-u.ac.jp

(cc) BY-NC-SA (02018 The Editorial Committee of Annals of Vascular Diseases. This article is distributed under the terms of the Creative Commons Attribution License, which permits use, distribution, and reproduction in any medium, provided the credit of the original work, a link to the license, and indication of any change are properly given, and the original work is not used for commercial purposes. Remixed or transformed contributions must be distributed under the same license as the original. ported more frequently after TEVAR than after open repair. ${ }^{1,2)}$ We report a case of delayed paraplegia triggered by gastrointestinal (GI) bleeding 8 months after TEVAR.

\section{Case Report}

A 78-year-old man with severe chronic obstructive pulmonary disease, hypertension, hyperlipidemia, and severe aortic atherosclerosis underwent TEVAR (RELAY Plus ${ }^{\circledR}$ stent graft, Bolton Medical, Barcelona, Spain: 40-145 mm, $44-200 \mathrm{~mm}$ ) for a descending true thoracic aneurysm (maximum diameter, $62 \mathrm{~mm}$ ). His renal function was normal. He had a history of smoking. During the perioperative period, mean blood pressure was maintained above $90 \mathrm{mmHg}$, with his hemoglobin above $12 \mathrm{~g} / \mathrm{dL}$ for spinal cord protection. Cerebrospinal fluid drainage was not employed. The stent graft extended from Th4 to L1 and covered the whole descending aorta. No neurological deficits developed during hospitalization, and he was discharged on postoperative day 8. Computed tomography (CT) at discharge showed patent subclavian, internal, external iliac, and hypogatric arteries. His postoperative course was uneventful in the outpatient setting until 8 months postoperatively, when he developed sudden dizziness and returned to our hospital. He was ambulatory and conscious, with a hemoglobin $(\mathrm{Hgb})$ concentration of $2.6 \mathrm{~g} / \mathrm{dL}$ and systolic blood pressure of $70-80 \mathrm{mmHg}$. His $\mathrm{Hgb}$ concentration 2 weeks prior was $8.6 \mathrm{~g} / \mathrm{dL}$. Immediate intravenous fluid bolus improved his systolic blood pressure to $90-100 \mathrm{mmHg} 2 \mathrm{~h}$ after admission. His $\mathrm{Hgb}$ concentration improved to $7.8 \mathrm{~g} / \mathrm{dL} 5 \mathrm{~h}$ after admission with 6 units of blood transfusion. Emergent esophagogastroduodenoscopy after hemodynamic recovery revealed a bleeding gastric ulcer that was almost hemostatic and required no intervention. He had no previous history of gastrointestinal bleeding, and he received neither aspirin nor gastric acid suppressants. His bilateral lower-extremity weakness was recognized $20 \mathrm{~h}$ after admission. The exact timing of the onset of paraplegia was unknown. During those $20 \mathrm{~h}$, his systolic blood pressure and hemoglobin remained above $130 \mathrm{mmHg}$ and $10 \mathrm{~g} / \mathrm{dL}$, 
a)

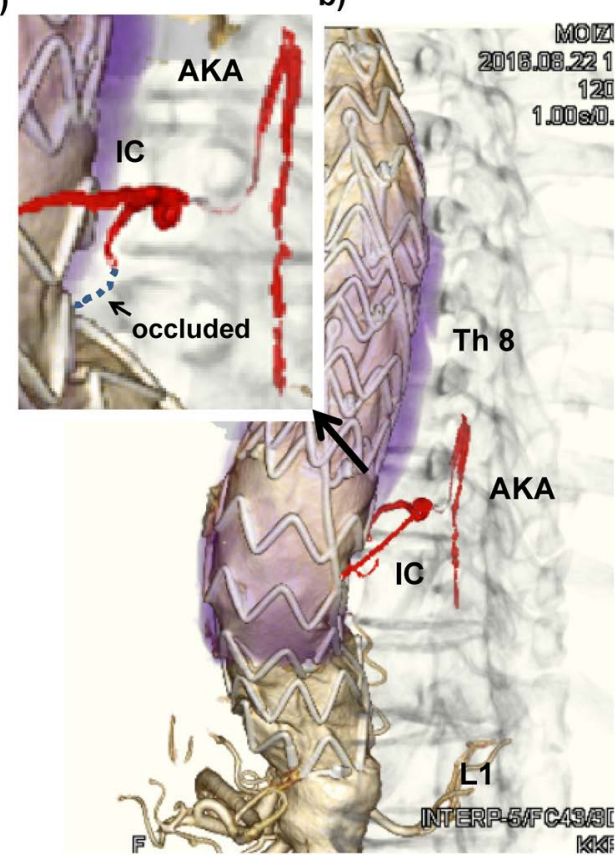

c)

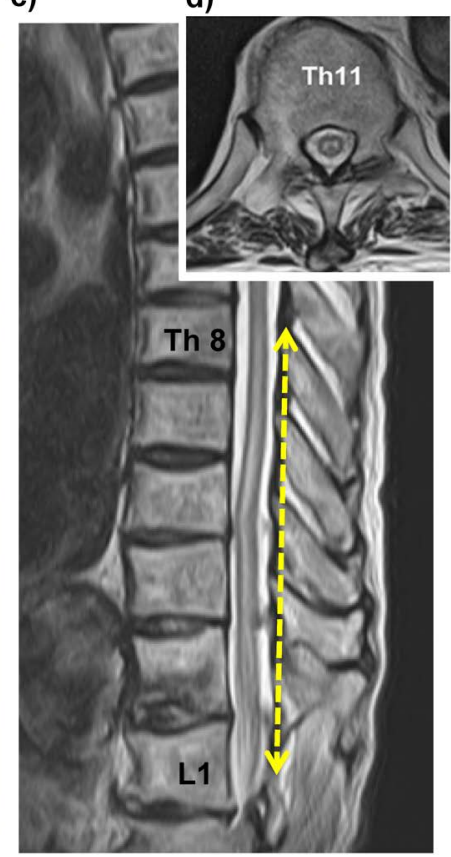

Fig. 1 (a-b) Enhanced computed tomography showed patent Adamkiewicz artery (AKA) connected with left Th11 intercostal artery, whose ostium at the descending aorta was occluded. (c-d) MRI showed spinal cord infarction from Th8 to L1.

AKA: Adamkiewicz artery; IC: intercostal artery; MRI: magnetic resonance imaging

respectively. Enhanced CT showed a patent Adamkiewicz artery (AKA) connected to the left Th11 intercostal artery, and the ostium at the descending aorta was occluded (Figs. 1a and 1b). Emergent magnetic resonance imaging (MRI) demonstrated a spinal cord infarction from Th8 to L1 (Figs. 1c and 1d). Cerebrospinal fluid drainage and medical support to maintain elevated blood pressure with vasopressor and fluid infusion did not improve his motor function. He had pain sensation with relative sparing of the proprioception and vibratory senses, suggesting anterior spinal artery syndrome.

\section{Discussion}

Spinal cord ischemia (SCI) is rare. Its prevalence is not well known, and it has a poor prognosis. It accounts for approximately $1 \%-2 \%$ of all vascular neurologic pathologies. ${ }^{3)}$ Several causes are implicated in SCI, including embolic disease, dissection, systemic hypotension, aortic operations, and idiopathic causes. ${ }^{4)}$ The location of SCI varies from the cervical to lumbar regions.

The proposed mechanism of the neurological deficit in this case is associated with the change in the blood supply to the AKA after TEVAR. The ostium of the intercostal artery of the descending aorta connected with the AKA was covered with the stent graft, which likely reduced the blood flow reserve for the AKA. Systemic hypotension and anemia due to GI bleeding might have reduced the blood supply to the AKA, resulting in SCI around the level of the AKA, which was demonstrated by MRI.

This patient might have developed paraplegia from GI bleeding even without a history of TEVAR. Moreover, it is unclear whether SCI occurs more frequently because of hypotension or anemia in patients with a previous history of TEVAR than in those without. However, decrease in blood flow reserve for the AKA is theoretically possible after TEVAR, especially in patients with severe atherosclerosis or with a previous history of other aortic surgical procedures, whose collateral network of blood supply to the spinal cord is reduced. If hypotension and anemia were the only causes of SCI in this case, the ischemic changes might have occurred at watershed areas, such as C4-7 instead. ${ }^{5)}$ The present clinical course combined with MRI and CT findings might provide evidence of vulnerability to SCI around the AKA after TEVAR, which has been considered but was difficult to demonstrate in the real-life setting. Physicians should bear in mind that patients with a previous history of TEVAR might be more vulnerable to SCI, which may be triggered by common hemorrhagic diseases, such as GI bleeding, even months or years after TEVAR. Patients with a history of TEVAR that covers a wide range of the descending thoracic aorta might require periodic examinations to check for GI bleeding. However, we could not demonstrate the exact values of blood pres- 
sure, hemoglobin, and duration of hemodynamic instability that sufficiently lead to SCI only from the present case. Moreover, it would be interesting to know the exact timing of the onset of neurological deficit (during or after the recovery of hypotension and anemia). If paraplegia developed after the recovery of the hypotension and anemia, the phenomenon might be close to so-called delayed paraplegia, and we should continue to pay attention to patients' lower limb activity after the recovery of anemia and hemodynamic instability. Further research is required to elucidate the mechanisms, risk factors, and treatment strategies of the development of spinal cord ischemic damage long after TEVAR.

\section{Conclusion}

We should bear in mind that vulnerability to SCI after TEVAR possibly persists in a chronic phase, especially for patients with severe atherosclerosis or with a previous history of other aortic surgical procedures. During the follow-up period after TEVAR, physicians should also pay attention to patients' general status, such as anemia or symptom of gastrointestinal bleeding, in order to prevent hypotension or hypoxia.

\section{Disclosure Statement}

All authors have no conflict of interest.

\section{Author Contributions}

\section{Writing: TY}

Critical review and revision: all authors

Final approval of the article: all authors

Accountability for all aspects of the work: all authors

\section{References}

1) Maeda T, Yoshitani K, Sato S, et al. Spinal cord ischemia after endovascular aortic repair versus open surgical repair for descending thoracic and thoracoabdominal aortic aneurism. J Anesth 2012; 26: 805-11.

2) Ullery BW, Cheung AT, Fairman RM, et al. Risk factors, outcomes, and clinical manifestations of spinal cord ischemia following thoracic endovascular aortic repair. J Vasc Surg 2011; 54: 677-84.

3) Sandson TA, Friedman JH. Spinal cord infarction. Report of 8 cases and review of the literature. Medicine (Baltimore) 1989; 68: 282-92.

4) Estrera AL, Miller CC 3rd, Huynh TT, et al. Preoperative and operative predictors of delayed neurologic deficit following repair of thoracoabdominal aortic aneurysm. J Thorac Cardiovasc Surg 2003; 126: 1288-94.

5) Vargas MI, Gariani J, Sztajzel R, et al. Spinal cord ischemia: practical imaging tips, pearls, and pitfalls. AJNR Am J Neuroradiol 2015; 36: 825-30. 